\title{
Model of correlated sequential adsorption of colloidal particles
}

\author{
R. Pastor-Satorras ${ }^{1}$ and J. M. Rubi ${ }^{2}$ \\ ${ }^{1}$ Department de Física i Enginyeria Nuclear, Universitat Politècnica de Catalunya, Campus Nord, Mòdul B4, 08034 Barcelona, Spain \\ ${ }^{2}$ Department de Física Fonamental, Facultat de Física-CER Física Sistemes Complexos, Universitat de Barcelona, Av. \\ Diagonal 647, 08028 Barcelona, Spain
}

(Received 5 February 2001; published 11 June 2001)

\begin{abstract}
We present results of a model of sequential adsorption in which the adsorbing particles are correlated with the particles attached to the substrate. The strength of the correlations is measured by a tunable parameter $\sigma$. The model interpolates between free ballistic adsorption in the limit $\sigma \rightarrow \infty$ and a strongly correlated phase, appearing for $\sigma \rightarrow 0$ and characterized by the emergence of highly ordered structures. The phenomenon is manifested through the analysis of several magnitudes, as the jamming limit and the particle-particle correlation function. The effect of correlations in one dimension manifests in the increased tendency to particle chaining in the substrate. In two dimensions the correlations induce a percolation transition, in which a spanning cluster of connected particles appears at a certain critical value $\sigma_{c}$. Our study could be applicable to more general situations in which the coupling between correlations and disorder is relevant, as for example, in the presence of strong interparticle interactions.
\end{abstract}

DOI: 10.1103/PhysRevE.64.016103

PACS number(s): 05.70.Ln, 68.43.-h

\section{INTRODUCTION}

The study of the irreversible adsorption of colloidal particles onto a surface has attracted a great deal of interest in the last years, due to its many practical applications in physics, chemistry, biophysics, medicine, etc. $[1,2]$. The understanding of these processes has been deepened mainly through the formulation of different models, defined via a set of rules by which the particles accommodate upon arriving at the surface. The different rules are responsible for the different values of the quantities describing the geometry of the absorbed phase, as the maximum fraction of the surface covered by particles-the jamming limit $\theta_{\infty}$ - or the particleparticle pair correlation function $g(r)$. In the random sequential adsorption model (RSA) [3-7] particles are placed at randomly selected positions on the surface. When an incoming particle overlaps with a previously adsorbed one, it is rejected; otherwise, it becomes irreversibly adsorbed. The RSA model is thus a good approximation when particles arrive at the surface purely by diffusion, and excluded volume effects are predominant [6]. In the ballistic model (BM) [8-11], on the other hand, particles descend to the surface following straight vertical trajectories. An incoming particle that does not reach the surface directly is allowed to roll over the previously adsorbed ones, following the steepest descent path, until it reaches a stable position. Particles that eventually rest on the surface are irreversibly adsorbed; otherwise they are rejected. The BM is thus a valid approximation to describe adsorption in the presence of very strong interactions between particles and substrate [12-14].

In the models described above, and in many of the variations of them analyzed so far, particles are supposed to interact only through short-range interactions-hard-core repulsion. The main exceptions are the analysis of the role played by electrostatic [15] and dipolar [14] interactions. In the absence of long-range interactions, the particles arriving at the surface are essentially uncorrelated from the adsorbed phase, and interact with them only via excluded-volume ef- fects. Therefore little is known about the general effect of correlations among the adsorbed phase and the adsorbing particles [16].

Our purpose in this paper is to analyze the influence of correlations among particles in the structure of the adsorbed phase in a simple numerical model. In our model, particles adsorb sequentially onto the surface. The presence of correlations affects the position on the surface in which the particles try to adsorb. In order to mimic the effect of long-range interactions, which tend to attract the incoming particles in the vicinity of the particles already attached, the trial position of the next particle is selected to be the position of a particle in the substrate plus an increment $\xi$, selected at random from a given probability distribution $\rho(\xi)$. Once the trial position has been selected, the adsorption process proceeds according to the rules of the BM model. The distribution $\rho(\xi)$ depends on a parameter $\sigma$ that interpolates between a flat distribution and a delta function, which allows us to explore the range between the absence of correlations and a very strong correlation effect, respectively. Apart from the obvious relation with the adsorption in the presence of long-range attractive interactions [14], the inclusion of general correlations in adsorption phenomena is relevant in other kinds of related problems, such as car parking, bird nesting, or adsorption with memory effects, where the state of the adsorbed phase at a given time exerts a strong influence on the position of the next incoming unit. We want to note that our model differs from the generalized ballistic-deposition models presented in Refs. [17] and [18] due to the implicit role as nucleation centers played in our model by the adsorbed particles.

The paper is organized as follows. In Sec. II we introduce the model in a general context, whereas in Sec. III we present the results of numerical simulations in dimensions $d=1$ and 2. Finally, in the last section we discuss the common features induced by correlations in the structure and geometry of the adsorbed phase. 


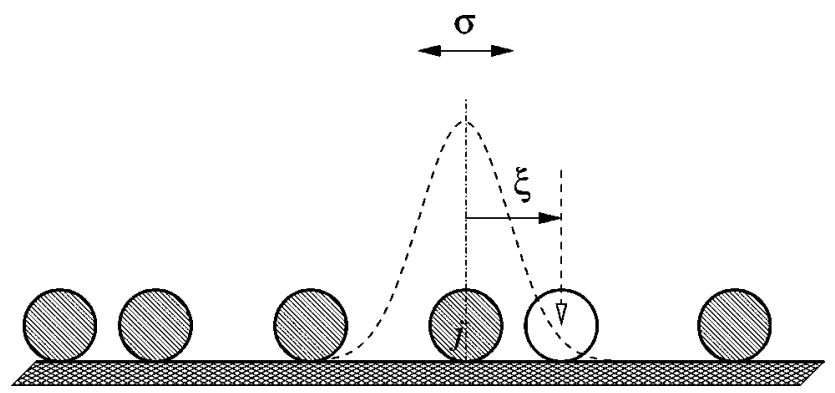

FIG. 1. Adsorption rule for the correlated adsorption model. An adsorbed particle $j$ is randomly selected. The newly adsorbed particle is deposited at a distance $\xi$ from the center of the selected particle. If it does not overlap with any other adsorbed particle, it is irreversibly attached. Otherwise, it is adsorbed following the prescription of the BM deposition.

\section{MODEL}

Our model considers the sequential adsorption of particles of diameter $a$ onto a $d$-dimensional surface; it is thus $d+1$ dimensional, including the direction perpendicular to the plane in which the particles move before adsorption. Particles arrive at the surface following straight vertical trajectories, and upon touching the surface or any adsorbed particle, they are attached following the rules of the BM. The key ingredient in the model, that differs from previous works, is the selection of the landing point of the arriving particles. In order to introduce correlations among the incoming particles and the adsorbed phase, the landing point is selected as follows: The first particle is located at random on the substrate. Suppose that, at a given time step, the substrate is composed by a set of $N$ particles, located at positions $\mathbf{R}_{i}$, $i=1, \ldots N$. The landing position of the next particle is chosen as

$$
\mathbf{R}^{\prime}=\mathbf{R}_{j}+\hat{\mathbf{n}} \xi
$$

where $\mathbf{R}_{j}$ is the position on the surface of a particle $j$ randomly selected among the $N$ particles present on the adsorbed phase, $\hat{\mathbf{n}}$ is a unity vector, parallel to the adsorption plane and oriented at random, and the distance $\xi$ is a Gaussian random variable, distributed according to the density

$$
\rho(\xi)=\frac{1}{\sqrt{2 \pi} a \sigma} \exp \left(-\frac{\xi^{2}}{2 a^{2} \sigma^{2}}\right),
$$

see Fig. 1. The only parameter in the model is $\sigma$, defined as the root-mean-square distance from an adsorbed particle at which the new particle is probed. Once the position $\mathbf{R}^{\prime}$ is selected, the particle is attached following the rules of the BM. If the particle is accepted, the updated position $\mathbf{R}^{\prime \prime}$ becomes the new value $\mathbf{R}_{N+1}$. If the particle is rejected, a new position is chosen and the procedure iterated.

The above algorithm has a very intuitive interpretation. Each one of the particles in the substrate plays the role of a nucleation center, attracting incoming particles towards them. We can envision this process as the result of longrange attractive interactions between the elements of the ad- sorbed phase and the incoming particles. The attraction has a random component given by the Gaussian term in Eq. (1), which represents the effect of the forces exerted on the incoming particle by the regions of the substrate located far away from the landing point. The strength of the attraction is given by the parameter $\sigma$ in Eq. (2). With this parameter we can interpolate between absolute lack of correlations, achieved in the limit $\sigma \rightarrow \infty$, and the case of very strong correlations, in the limit $\sigma \rightarrow 0$. In the former case, the Gaussian (2) tends to a flat distribution, which corresponds to a free BM adsorption. On the other hand, in the limit $\sigma$ $\rightarrow 0$ the distribution (2) becomes a sharply peaked delta function. In this case the correlations are maximal, and one would expect to obtain a close-packed substrate, at least in $1+1$ dimensions.

In the following section we report the result obtained from simulations performed with the random increment distribution given by Eq. (2). Other sets of simulations, done with a different peaked distribution [such as an exponential $\rho(\xi)=\exp (-\xi / a \sigma) / a \sigma]$, yield qualitatively similar results, confirming the fact that the important point is the presence of correlations, and not the particular form.

\section{RESULTS}

In order to check the effects of correlations in the adsorption process, we have performed extensive numerical simulations of our model in $1+1$ and $2+1$ dimensions. Simulations were done in systems of different size $L$. Statistical averages were performed over at least 100 different realizations. In order to avoid cross effects between the characteristic length $a \sigma$ and the system size $L$, we always consider $a \sigma \ll L$.

\section{A. Adsorption in $1+1$ dimensions}

We have performed simulations of the correlated adsorption model in $1+1$ dimensions over a substrate consisting of a line of length $L=5000 a$, with periodic boundary conditions.

In $1+1$ dimensions, the most relevant feature of the model is the increased tendency of the adsorbed particles to form connected structures, identified with chains, whose average length tends to increase with decreasing $\sigma$. We can use as a measure of this kind of order the chain-length density function, $P(l)$, defined as the average number of chains of length $l a$ per unit length of substrate. In Fig. 2 we represent the chain-length density function computed at different values of $\sigma$. The value $\sigma=\infty$ corresponds to simulations of free BM adsorption. For large $\sigma$ we observe a very fast (superexponential) decay of $P(l)$, indicative of a lack of any characteristic length scale. For small values of $\sigma$, on the other hand, $P(l)$ shows a clear exponential tail, $P(l) \sim \exp \left(-l / l_{c}\right)$, with a characteristic length $l_{c}$ depending on $\sigma$. In particular, for $\sigma=1$ we estimate a value $l_{c}=2.48 \pm 0.05$.

Another quantity of interest is the jamming limit $\theta_{\infty}$, which in the present case can be related to the chain-length density function through the expression 


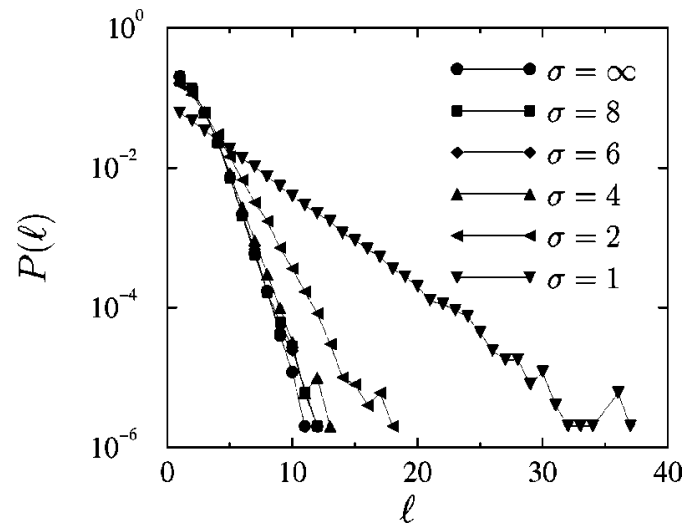

FIG. 2. Chain-length density function $P(l)$ for different values of the parameter $\sigma$ in $(1+1)$-dimensional correlated adsorption.

$$
\theta_{\infty}=\sum_{l=1}^{\infty} l P(l) .
$$

In Fig. 3 we represent the jamming limit as a function of the parameter $\sigma$. For values of $\sigma$ larger than about 8 we recover with good accuracy the limit of free BM adsorption, $\theta_{\infty}^{\mathrm{BM}}$ $\simeq 0.80865$ [9]. For smaller values of $\sigma$, on the other hand, the presence of the correlations induces a higher compaction on the substrate, with a jamming limit that approaches the close packing for a value of $\sigma$ equal to $0.5\left(\theta_{\infty}=0.9918\right.$ $\pm 0.0005)$.

As a final probe of the substrate's structure, we have measured the particle-particle pair correlation function $g(r)$. In Fig. 4 we plot $g(r)$ as a function of the reduced distance $r / a$ corresponding to six different values of $\sigma$. We observe that the correlations enhance the maxima of $g(r)$, which occur at distances $p a$ for integer $p$ (corresponding to interparticle distances equal to a multiple of the diameter). The decay of the maxima is close to exponential again. For large values of $\sigma$ we recover the behavior of the free BM adsorption, with peaks decaying superexponentially.

\section{B. Adsorption in $2+1$ dimensions}

Simulations in $2+1$ dimensions were performed on squares of size $L=125 a$, with periodic boundary conditions.

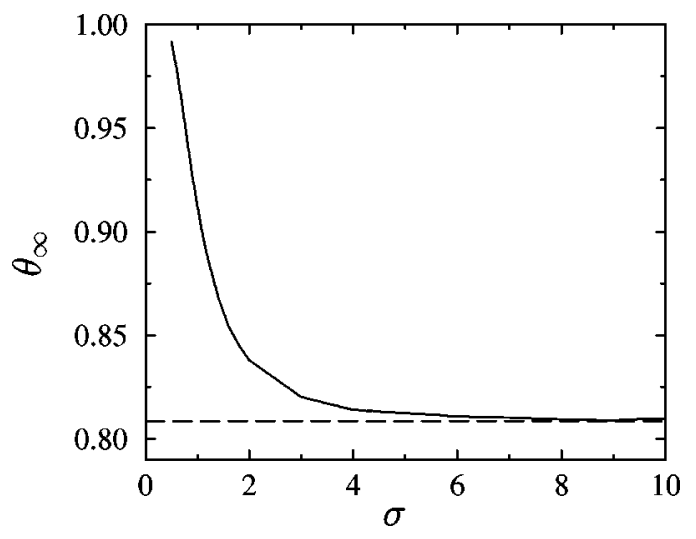

FIG. 3. Jamming limit $\theta_{\infty}$ as a function of the parameter $\sigma$ in $(1+1)$-dimensional correlated adsorption. The dashed line signals the jamming limit for free $\mathrm{BM}$ adsorption, $\theta_{\infty}^{\mathrm{BM}} \simeq 0.80865$.

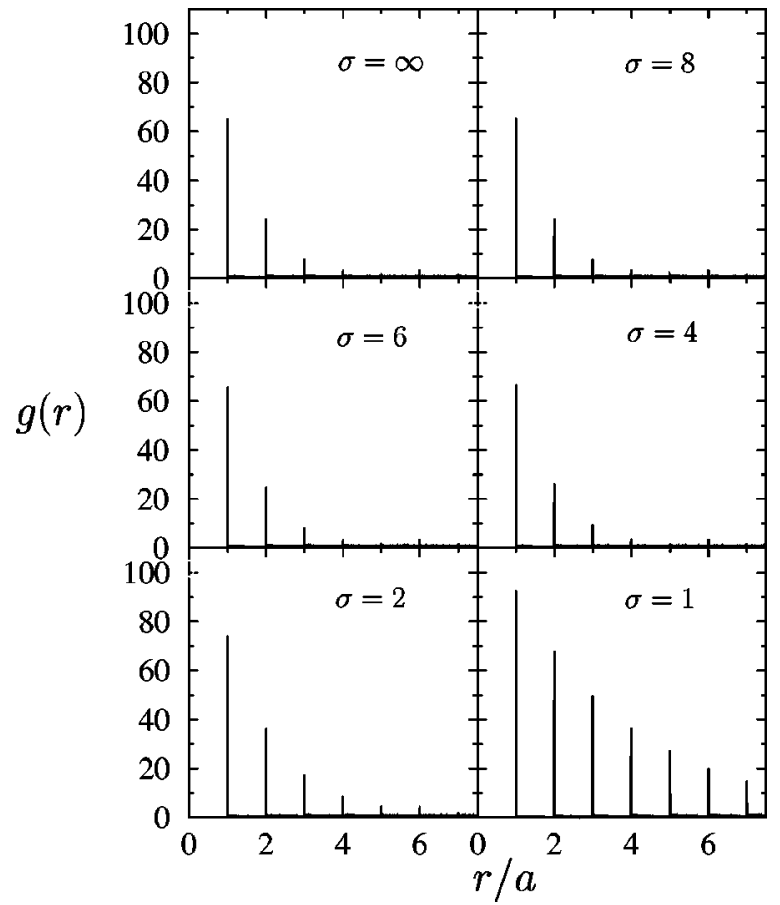

FIG. 4. Particle-particle correlation function $g(r)$ for different values of $\sigma$ in $(1+1)$-dimensional correlated adsorption.

In Fig. 5 we plot the jamming limit $\theta_{\infty}$ obtained from simulations for different values of $\sigma$. The trend previously observed in $1+1$ dimensions is here recovered. For large values of $\sigma$ the jamming limit tends to the free BM adsorption $\theta_{\infty}^{\mathrm{BM}} \simeq 0.610$ [11]. For decreasing $\sigma, \theta_{\infty}$ increases approaching the close-packing regime; for $\sigma=0.5$, for example, we estimate $\theta_{\infty}=0.715 \pm 0.001$ [19]. It should be noted that the convergence of $\theta_{\infty}$ towards its maximum value is slower in this case than in the adsorption in $1+1$ dimensions (compare Figs. 3 and 5).

In Fig. 6 we plot the particle-particle correlation function $g(r)$ corresponding to the $2+1$ case. For $\sigma=\infty$, we recover the expected form in free BM adsorption (the divergent peak at $r=a$, corresponding to the close contact of particles, has been omitted for the sake of clarity). The secondary peak at

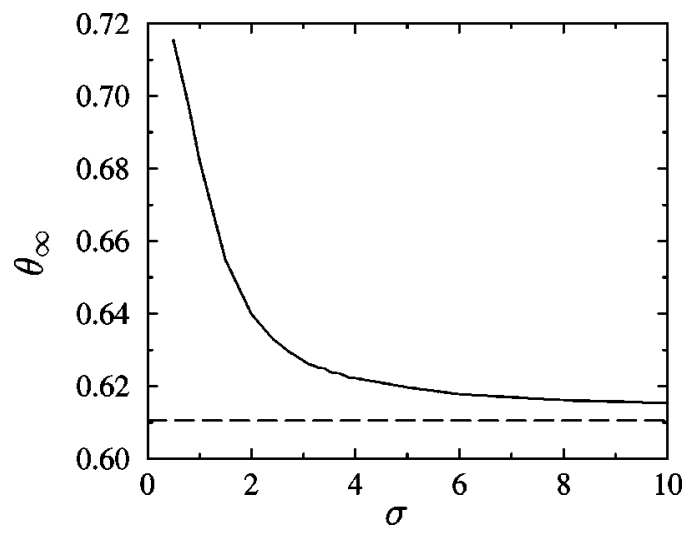

FIG. 5. Jamming limit $\theta_{\infty}$ as a function of the parameter $\sigma$ in $2+1$-dimensional correlated adsorption. The dashed line signals the jamming limit for free $\mathrm{BM}$ adsorption, $\theta_{\infty}^{\mathrm{BM}} \simeq 0.610$. 


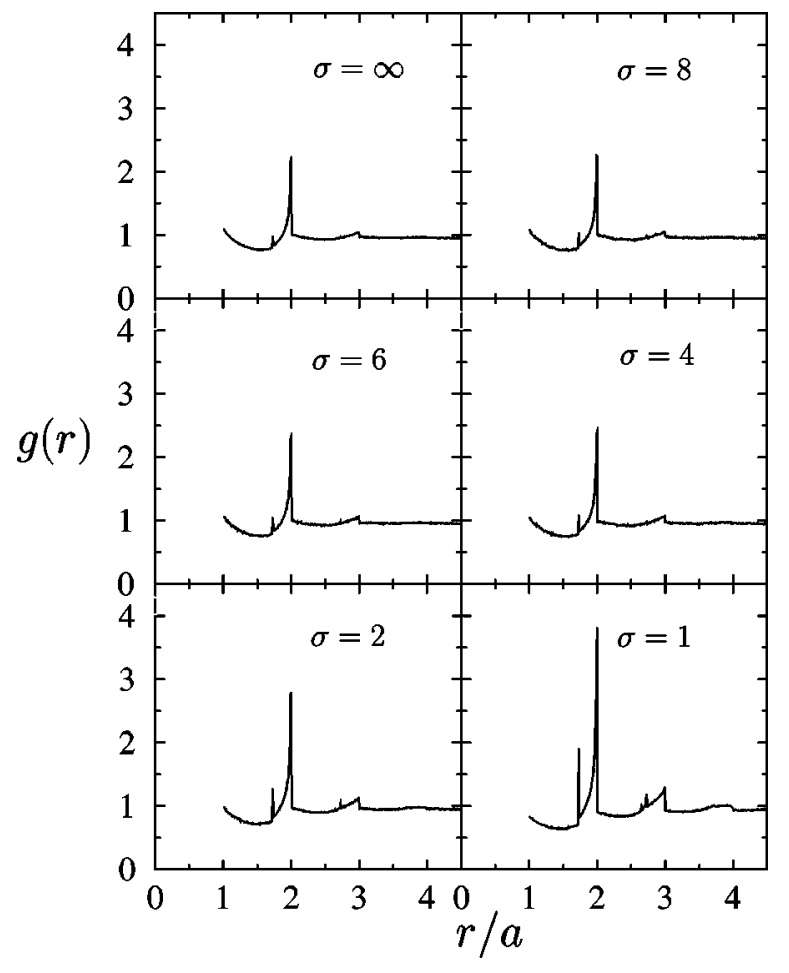

FIG. 6. Particle-particle correlation function $g(r)$ for different values of $\sigma$ in $(2+1)$-dimensional correlated adsorption. The divergent peak at $r=a$ has been suppressed for clarity.

$r=2 a$ is clearly visible, as well as an intermediate, much smaller peak, located approximately at $r \simeq 1.73 a$. For decreasing $\sigma$ the height of the secondary peak increases notably, and the intermediate peak becomes more and more noticeable.

From the analysis of the correlation function we can conclude that the correlations in the adsorption mechanism induce the creation of connected structures on the substrate, which are responsible for the enhancement of the peaks in $g(r)$. These connected structures cannot be characterized in terms of chains, since in $2+1$ dimensions particles adsorb in multiply connected configurations, and therefore the notion of chain loses its meaning. In this case, however, the struc-

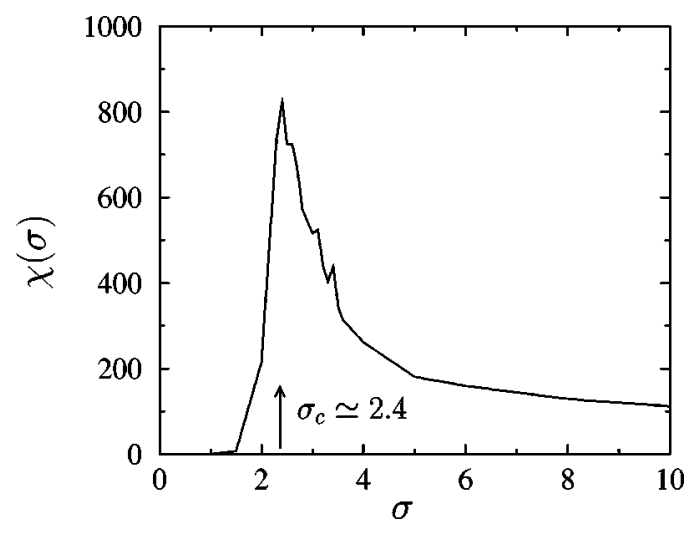

FIG. 7. Susceptibility for the $(2+1)$-dimensional correlated adsorption. The peak, located at $\sigma_{c} \simeq 2.4$, signals the presence of a percolation transition.
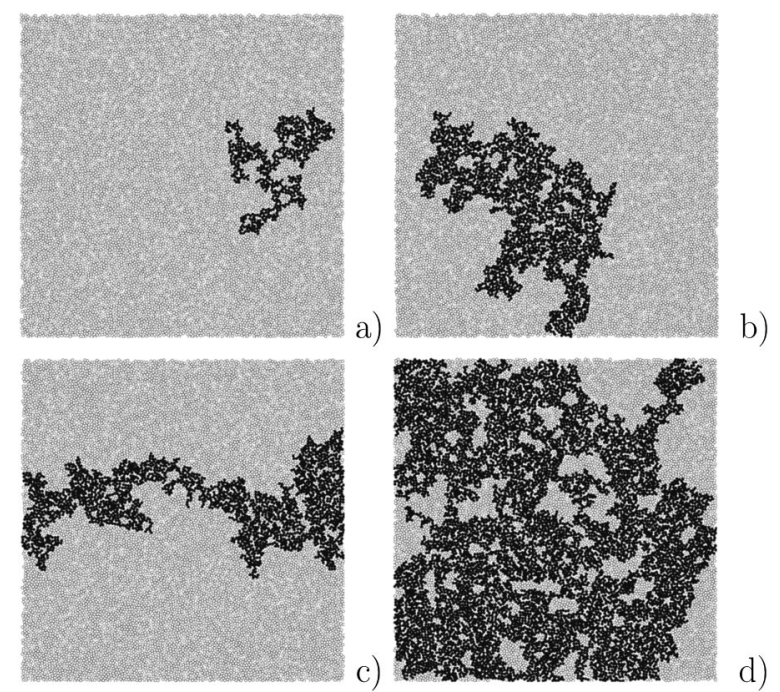

b)

FIG. 8. Largest cluster of connected particles in (2+1)-dimensional correlated adsorption. (a) $\sigma=\infty$; (b) $\sigma=2.8$; (c) $\sigma=2.4$, (d) $\sigma=2.0$.

ture of the adsorbed phase can be better analyzed in terms of its percolation properties $[20,18,21]$. Since the particles can roll one above the other before adsorption, the substrate becomes eventually composed by clusters of connected particles, which can be easily identified. In free BM adsorption, the saturated phase has low connectivity and it is below the percolation threshold: in simulations on any finite system of size $L$, the largest cluster of connected adsorbed particles is not a spanning cluster. In order to investigate the percolation properties of the correlated adsorption model, we have computed the susceptibility $\chi$ of the distribution of clusters of connected particles as a function of $\sigma$. The susceptibility is defined by [22]

$$
\chi=\sum_{s}^{\prime} n_{s} s^{2}
$$

where $n_{s}$ denotes the density of clusters of size $s$ and the prime in the summation indicates that the biggest (spanning) cluster is excluded in the sum. At the percolation threshold $\sigma_{c}$, the susceptibility is expected to exhibit a peak, with power-law decay both below and above the threshold. In Fig. 7 we have plotted the susceptibility $\chi$ computed as a function of the parameter $\sigma$. The presence of a maximum in $\chi$, located approximately at $\sigma_{c} \simeq 2.4$, signals the presence of a percolation transition. Below the threshold, the clusters of connected particles are numerous and rather small. Above the threshold $\left(\sigma<\sigma_{c}\right)$, however, there exists a spanning cluster, that crosses the system from boundary to boundary. We show the presence of such a spanning cluster in Fig. 8. For $\sigma>2.4$, the largest cluster in a typical simulation of our model is rather small. For $\sigma=2.4$ a largest cluster develops that first spans the whole system. For even smaller values of $\sigma$, the largest cluster covers most of the substrate.

\section{CONCLUSIONS}

In this paper we have presented a different model of correlated sequential adsorption of particles on a substrate 
which takes into account the existence of a certain degree of correlation between the final positions of the adsorbing particles and the location of the preadsorbed ones. Its implementation is carried out by modifying the rules of the BM, by imposing that the final position of an incoming particle is randomly distributed, with a specified probability density, around the location of a particle in the substrate. In this way, the model can describe many different physical situations ranging from the absence of correlations, corresponding to BM model, to the pure deterministic case in which the distribution of particles practically reaches the close-packing configuration.

The main consequence of the existence of correlations is the appearance of long-range order in the adsorbed phase. In $1+1$ dimensions, this feature manifests in the fact that the length of the chains increases when decreasing the variance of the probability distribution. In $2+1$ dimensions, we have reported the existence of a percolation transition which can be identified due to the presence of a peak in the susceptibility. In all cases, the jamming limit increases significantly when the probability distribution function becomes sharper, and is close, in the limit of a sharply peaked distribution, to the close-packing regime.

Our model could caricature some real situations, not addressed by the sequential adsorption models proposed up to now, sharing in common the fact that the final position of an adsorbed particle may exert an influence in the location of the next particle. Cases in which the particles interact, when the kinetics exhibits memory effects or, in general, when the adsorption process may be in some way controlled by an external agent and therefore is not totally random, could accordingly find a description in the model we have proposed. The results we have presented may provide new possibilities to the already existing ones about modelization of adsorption kinetics by sequential processes.

\section{ACKNOWLEDGMENTS}

R.P.S. acknowledges support from Grant No. CICyT PB97-0693. J.M.R. acknowledges support from Grant No. DGICyT PB98-1258.
[1] M.C. Bartelt and V. Privman, Int. J. Mod. Phys. B 5, 2883 (1991).

[2] J. Evans, Rev. Mod. Phys. 65, 1281 (1993).

[3] A. Rényi, Sel. Trans. Math. Stat. Prob. 4, 203 (1963).

[4] J. Feder, J. Theor. Biol. 87, 237 (1980).

[5] P. Schaaf and H. Reiss, J. Chem. Phys. 92, 4824 (1988).

[6] B. Senger, J.-C. Voegel, P. Schaaf, A. Johner, A. Schmitt, and J. Talbot, Phys. Rev. A 44, 6926 (1991).

[7] J.J. Ramsden, Phys. Rev. Lett. 71, 295 (1993).

[8] P. Meakin and R. Jullien, J. Phys. (Paris) 48, 1651 (1987).

[9] J. Talbot and S.M. Ricci, Phys. Rev. Lett. 68, 958 (1992).

[10] R. Jullien and P. Meakin, J. Phys. A 25, L189 (1992).

[11] A.P. Thompson and E.D. Glandt, Phys. Rev. A 46, 4639 (1992).

[12] B. Senger, R. Ezzeddine, F.J. Bafaluy, P. Schaaf, F.J.G. Cuisinier, and J.-C. Voegel, J. Theor. Biol. 163, 457 (1993).

[13] P. Schaaf, P. Wojtaszczyk, B. Senger, J.C. Voegel, and H. Reiss, Phys. Rev. E 51, 4292 (1995).

[14] R. Pastor-Satorras and J.M. Rubí, Phys. Rev. Lett. 80, 5373 (1998).
[15] Z. Adamczyk and P. Warszyński, Adv. Colloid Interface Sci. 63, 41 (1996).

[16] The BM in its original formulations, was referred to as "correlated sequential adsorption" (CSA) deposition, due to the very short-range correlations among particles induced by the sliding mechanism incorporated in the model [11]. In the present paper we are concerned with correlations of a more general nature, induced by external agents or interactions.

[17] P. Viot, G. Tarjus, and J. Talbot, Phys. Rev. E 48, 480 (1993).

[18] H.S. Choi, J. Talbot, G. Tarjus, and P. Viot, Phys. Rev. E 51, 1353 (1995).

[19] The maximum value of $\theta_{\infty}$ in $2+1$ dimensions will be smaller than the one corresponding to a close-packed substrate, due to the inherent randomness of our model.

[20] D. Stauffer and A. Aharony, Introduction to Percolation Theory, 2nd ed. (Taylor \& Francis, London, 1994).

[21] N. Vandewalle, S. Galam, and M. Kramer, Eur. Phys. J. B 14, 407 (2000).

[22] S.B. Lee, Phys. Rev. B 42, 4877 (1990). 